\title{
Identificación de Hongos Micorrízicos en Plantaciones de Melina (Gmelina arborea Roxb) en el Trópico Húmedo Ecuatoriano
}

\author{
Identification of Arbuscular Mycorrhizal Fungi in Melina (Gmelina arborea \\ Roxb) Plantations in the Ecuadorian Humid Tropics \\ Fabricio Meza B. ${ }^{1,2,5}$, Eduardo Díaz O. ${ }^{1}$, Harold Escobar T. ${ }^{1}$, Carlos Belezaca \\ Pinargote $^{1}$, Jesica Cachipuendo C. ${ }^{2}$, Gary Meza B. ${ }^{1,2}$, Francel López M. ${ }^{1,3}$, Carlos \\ Meza B. ${ }^{2}$, Jessica Meza B. ${ }^{2}$, Judith Cachipuendo C. ${ }^{4}$, Rodrigo Cabrera V. ${ }^{1}$
}

\section{Resumen}

El objetivo de esta investigación fue aislar e identificar hongos formadores de micorriza arbuscular en plantaciones de melina (Gmelina arborea Roxb). Se muestrearon dos plantaciones de melina de 1 y 3 años de edad, ubicadas en la zona central del trópico húmedo ecuatoriano, provincia de Los Ríos, cantón Valencia. Las muestras de suelo y raíces se recolectaron en la época seca entre junio y diciembre de 2015. Los resultados mostraron la presencia de los géneros de hongos micorrízicos arbusculares Glomus y Gigaspora. El género con mayor representatividad en cantidad de esporas por gramo de suelo fue Glomus. El mayor porcentaje de colonización micorrízica se presentó en las raíces de los árboles de 1 año de edad.

Palabras clave: Gmelina arborea Roxb; agroecosistemas; micorrizas arbusculares

\section{AbStract}

This study aimed to isolate and identify arbuscular mycorrhizal fungi in melina (Gmelina arborea Roxb) plantations. Two melina plantations of 1 and 3 years old located in the central zone of the humid Ecuadorian tropics, province of Los Ríos, Valencia were sampled. Soil and root samples were collected in the dry season between June and December 2015. The results showed the presence of the arbuscular mycorrhizal fungi

\footnotetext{
${ }^{1}$ Universidad Técnica Estatal de Quevedo, Los Ríos, Ecuador

${ }^{2}$ Instituto Tecnológico Superior Ciudad de Valencia, Los Ríos, Ecuador

${ }^{3}$ Universidad Tecnológica Equinoccial, Santo Domingo de los Tsachilas, Ecuador

${ }^{4}$ Gobierno Autónomo Descentralizado Municipal Pastaza

${ }^{5}$ E-mail:f-abri-67@hotmail.com
}

Recibido: 13 de marzo de 2017

Aceptado para publicación: 28 de julio de 2017 
genera Glomus and Gigaspora. The genus with the greatest representativeness in quantity of spores per gram of soil was Glomus. The highest percentage of mycorrhizal colonization occurred in the roots of 1-year-old trees.

Key words: Gmelina arborea Roxb; agroecosystems; arbuscular mycorrhiza

\section{INTRODUCCIÓN}

Los hongos micorrízicos arbusculares (HMA) son microorganismos rizosféricos simbióticos de más del $80 \%$ de las plantas (Smith y Read, 2008), localizados en las raíces de la mayoría de las comunidades vegetales de herbáceas y árboles tropicales. Estos hongos forman parte del orden Glomerales (Varela y Trejo, 2001). El efecto benéfico de los HMA (definido como efectividad) en la promoción del crecimiento y nutrición de las plantas parece estar definido por la riqueza de especies y por la procedencia de su aislamiento (Trejo et al., 2011). Aun cuando no son específicos, los HMA pueden presentar mayor compatibilidad hacia algunas especies vegetales (van der Heijden et al., 1998). Así, algunos HMA pueden estimular el crecimiento, y otros pueden favorecer la absorción de nutrientes, inducir resistencia a fitopatógenos, o ayudar en la adaptación y tolerancia de las plantas ante condiciones de estrés.

La identificación taxonómica de los HMA ha sido incipiente en el trópico húmedo ecuatoriano (THE), ya que la mayor parte de los estudios se han enfocado en determinar la respuesta de la planta a la micorriza, sin considerar la procedencia o identidad del endófito en plantaciones forestales de melina (Gmelina arborea). Este es un árbol caducifolio de la familia Lamiaceae, especie de rápido crecimiento, que se ha adaptado satisfactoriamente a las condiciones del THE, a tal punto que existen 2366 ha plantadas con esta especie (Reybampac, 2012).
Se conoce que especies forestales a nivel de vivero responden satisfactoriamente a inoculaciones de HMA, mostrando respuestas superiores a las variables dasométricas, frente a plántulas no inoculadas y, por tanto, favoreciendo su sobrevivencia en el campo (Donoso et al., 2008). Basado en este contexto, se planteó como objetivo aislar e identificar hongos formadores de micorriza arbuscular en plantaciones de melina.

\section{Materiales y Métodos}

La presente investigación se realizó en dos plantaciones forestales de melina (Gmelina arborea) de 1 y 3 años de edad, ubicadas en áreas de 10 y 8 ha, respectivamente, en una finca de la zona central del trópico húmedo ecuatoriano; específicamente en el cantón Valencia, Provincia de Los Ríos, Ecuador. Se consideró que las plantaciones forestales han sido conservadas con escaso disturbio del suelo y sin aplicación de pesticidas químicos. Los análisis de suelo y raíces se realizaron en el Laboratorio de Microbiología Ambiental y Vegetal y en el invernadero de la Universidad Técnica Estatal de Quevedo (UTEQ).

Las características edafoclimáticas dominantes de la zona de estudio fueron: suelos arenosos con topografía originados a partir de cenizas volcánicas. La temperatura promedio anual es de $25.4^{\circ} \mathrm{C}$, precipitación promedio anual de $1609 \mathrm{~mm}, 82 \%$ de humedad relativa y 923 horas luz/año. 
Las plantaciones estaban ubicadas una al lado de la otra, por lo que las condiciones de suelo fueron similares. Se tenían 1111 árboles/ha, con una distancia de siembra de $3 \times 3$ $\mathrm{m}$ en ambas plantaciones. Las muestras de suelo y raíces se recolectaron entre junio y diciembre (época seca) de 2015. Para la colecta de las muestras de suelos y raíces se utilizó una escarbadora, barreno y fundas de plásticos rotuladas siguiendo la metodología propuesta por Cuervo y Rivas (2016). Esto implica que cada sitio de muestreo está conformado por seis árboles y se toman dos submuestras de suelo y raíces por árbol (proyección de la copa sobre el suelo). Las muestras de cada sitio se mezclaron hasta obtener $2 \mathrm{~kg}$ de suelo y $80 \mathrm{~g}$ de raíces, lo cual permitió obtener muestras homogéneas de suelo y raíces hasta una profundidad de 20 $\mathrm{cm}$. Las muestras fueron preservadas en refrigeración (entre 4 y $10{ }^{\circ} \mathrm{C}$ ).

A los suelos procedentes de cada plantación estudiada se le midió el $\mathrm{pH}$ empleando la metodología propuesta por Steubing et al. (2001), que mide el $\mathrm{pH}$ en agua $\left(\mathrm{H}_{2} \mathrm{O}\right) \mathrm{y}$ cloruro de potasio $(\mathrm{KCl})$ al $0.1 \mathrm{~N}$. La lectura del pH se registró en el sobrenadante de las suspensiones con un pH-metro Oakton 510.

Para el aislamiento y conteo de esporas de HMA se utilizó el método de tamizado y decantación en húmedo con centrifugación, similar a la metodología propuesta por Gerderman y Nicholson (1963) con ciertas modificaciones. Se pesaron $100 \mathrm{~g}$ de suelo, las partículas de suelo fueron disgregadas con las manos y colocadas en un vaso de precipitación, donde se añadió $1000 \mathrm{ml}$ de agua. La suspensión se dejó reposar durante 2 min y se pasó a través de tamices con apertura de mallas de 425, 90 y $25 \mu \mathrm{m}$ para separar las esporas de acuerdo a su tamaño. La agitación y decantación se repitió tres veces.

El material que quedó atrapado en los tamices se vertió en vasos de precipitación de $100 \mathrm{ml}$ con $50 \mathrm{ml}$ de agua destilada. En tubos de centrífuga de $15 \mathrm{ml}$ se colocaron 5 $\mathrm{ml}$ de solución de sacarosa al 20\%. A partir de esa solución, se adiciono $5 \mathrm{ml}$ de sacarosa al $60 \%$ al fondo de la anterior para formar una gradiente de sacarosa. Por último, se agregaron $5 \mathrm{ml}$ de la solución del suelo suspendido en agua. Se sellaron los tubos con parafilm y se colocaron en la centrífuga durante 3 min a $1006 \mathrm{~g}$. El contenido del tamiz se recogió en envases de vidrio y fue vertido de a pocos en cajas Petri cuadriculadas de, aproximadamente, $1.25 \mathrm{~cm}$ entre líneas, para hacer el conteo de esporas en tres réplicas.

Para determinar la colonización micorrízica, las muestras de raíces $(80 \mathrm{~g})$ fueron lavadas con abundante agua corriente. Luego fueron cubiertas con una solución de hidróxido de potasio $(\mathrm{KOH})$ al $10 \%$ y se colocaron en baño de maría $\left(90^{\circ} \mathrm{C}\right)$ durante 10 a $15 \mathrm{~min}$. Posteriormente, las raíces se volvieron a lavar con agua corriente para eliminar el $\mathrm{KOH}$, utilizando un tamiz para evitar pérdidas durante el enjuague. Se pasó por un proceso de inmersión durante 5 a 10 min en una solución fresca de $\mathrm{KOH}$ al $10 \% \mathrm{y} \mathrm{H}_{2} \mathrm{O}_{2}$ al $10 \%$ en proporción 1:1 (V/V). Luego se lavó las raíces con agua corriente para pasar a un proceso de inmersión en ácido clorhídrico $(\mathrm{HCl})$ al $1 \mathrm{~N}$ durante $10 \mathrm{~min}$; se decantó el $\mathrm{HCl}$ y, sin lavar, se adicionó azul de tripano al $0.05 \%$ en lactoglicerol, colocando las raíces al baño de maría por $10 \mathrm{~min}$. Se volvió a lavar las raíces en agua destilada y se les dejó en reposo por $12 \mathrm{~h}$ para eliminar el exceso de colorante.

Para la determinación del porcentaje de colonización micorrízica en las raíces de melina, se realizaron montajes al microscopio. Se seleccionaron 50 segmentos de raíces adicionando gotas de glicerol $(50 \%)$. Se efectuaron tres repeticiones por plantación. Las observaciones se realizaron en un microscopio a 40x.

La frecuencia (\%) de colonización radicular se determinó considerando los segmentos colonizados y no colonizados. Se obtuvo la relación total de segmentos colonizados con respecto a los segmentos totales evaluados, y se contabilizó con base a la colonización total por arbúsculos y por vesículas. 
La identificación de los HMA a nivel de género se efectuó con la ayuda de claves taxonómicas (INVAM, sf; Brundrett et al., 1996; Peterson et al., 2004). El porcentaje de colonización micorrízica se evaluó mediante el método de despigmentación y tinción de raíz propuesta por Phillips y Hayman (1970) y Giovanotti y Mosse (1980).

Para identificar y evaluar el número de esporas de HMA por gramo de suelo húmedo $\left(\mathrm{gsh}^{-1}\right)$ se realizó un análisis descriptivo, mientras que para evaluar el porcentaje de colonización micorrízica en las plantaciones de melina se consideraron los segmentos colonizados y los no colonizados. Los datos obtenidos se analizaron utilizando los procedimientos ANOVA de $\mathrm{SAS}{ }^{\circledR}$ v. 9.0 para Windows (SAS/STAT $\left.{ }^{\circledR}, 2002\right)$. Se empleó un diseño completamente al azar. Las diferencias estadísticas entre los promedios de los tratamientos fueron determinadas mediante la prueba de Tukey $(\mathrm{p}<0.05)$.

\section{Resultados y Discusión}

La plantación de 1 año de edad fue la que presentó el mayor número de esporas por $\mathrm{gsh}^{-1}$. Con relación al tamaño de los tamices, el número de esporas fue significativamente diferente entre la plantación de 1 año y la plantación de 3 años con el tamiz de $90 \mu \mathrm{m}$ (23 vs 19 esporas, respectivamente (Cuadro 1 ).

Las esporas de HMA correspondieron a los géneros Glomus y Gigaspora (Cuadro 2). Estos géneros de hongos son similares a los reportados por Prieto-Benavides et al. (2012), en sistemas agroforestales de cacao, quiénes además encontraron hongos de los géneros Acaulospora y Scutellospora. Por otro lado, Méndez-Cortés et al. (2103) identificaron los géneros Glomus, Funneliformis, Rhizophagus, Acaulospora y Pacispora para selva alta perennifolia (SAP) y Claroideoglomus, Funneliformis, Glomus, Rhizophagus, Sclerocystis, Paraglomus,
Cuadro 1. Población total de esporas HMA por cada $100 \mathrm{~g}$ de suelo húmedo $\left(\mathrm{gsh}^{-1}\right)$ en dos plantaciones de melina (Gmelina arborea) del trópico húmedo ecuatoriano, según edad de los árboles (2016)

\begin{tabular}{ccc}
\hline $\begin{array}{c}\text { Apertura de } \\
\text { tamiz }\end{array}$ & 1 año & 3 años \\
\hline $425 \mu \mathrm{m}$ & $19^{\mathrm{a}}$ & $17^{\mathrm{a}}$ \\
$90 \mu \mathrm{m}$ & $23^{\mathrm{a}}$ & $19^{\mathrm{b}}$ \\
$25 \mu \mathrm{m}$ & $28^{\mathrm{a}}$ & $26^{\mathrm{a}}$ \\
\hline Total & 70 & 62 \\
\hline a,b Superíndices diferentes dentro de filas indica \\
\multicolumn{2}{l}{ diferencia significativa ( $\mathrm{p}<0.05)$}
\end{tabular}

Entrophospora, Scutellospora y Pacispora para selva alta superennifolia (SMS).

La mayor cantidad de esporas se atrapó en el tamiz de $25 \mu \mathrm{m}$, seguido de los tamices de 90 y $425 \mu \mathrm{m}$ (Cuadro 2). Se encontró un número significativamente mayor de esporas del género Glomus en las muestras de la plantación de 1 año de edad (45 esporas) que en las muestras de la plantación de 3 años de edad (33 esporas) $(\mathrm{p}<0.05$; Cuadro 2).

La diversidad de géneros de hongos formadores de MA encontrados en las plantaciones de melina, tienen algo en común y distan en cuanto a géneros de HMA localizados en ecosistema nativos, como lo señala Ruíz et al. (2011) en estudios realizados en bosques de la amazonia peruana, donde encontraron Paraglomus occulatum y Glomus microaggregatum. Hasta ahora no se sabe qué factores influyen en la diversidad y la composición de las especies de HMA, pudiendo ser el tipo de suelo, las condiciones climáticas y la composición florística de cada lugar en particular (Ruíz et al., 2011). No obstante, se ha demostrado que hay diferentes comunidades de HMA en distintos hábitats y que en un mismo hábitat puede haber diferente composición de HMA en cada especie 
Cuadro 2. Número de esporas y género de hongos formadores de micorriza arbuscular identificados y obtenidos por cada 100 gramos de suelo húmedo $\left(\mathrm{gsh}^{-1}\right)$ en tres tamaños de tamices en plantaciones de melina (Gmelina arborea) ubicados en la zona central del trópico húmedo ecuatoriano (2016)

\begin{tabular}{lcccccccc}
\hline \multirow{2}{*}{ Género } & \multicolumn{9}{c}{1 año } & \multicolumn{5}{c}{3 años } \\
\cline { 2 - 9 } & $425 \mu \mathrm{m}$ & $90 \mu \mathrm{m}$ & $25 \mu \mathrm{m}$ & Total & $425 \mu \mathrm{m}$ & $90 \mu \mathrm{m}$ & $25 \mu \mathrm{m}$ & Total \\
\hline Glomus & 12 & 15 & 18 & $45^{\mathrm{a}}$ & 9 & 10 & 14 & $33^{\mathrm{b}}$ \\
Gigaspora & 7 & 8 & 10 & $25^{\mathrm{a}}$ & 8 & 9 & 12 & $29^{\mathrm{a}}$ \\
\hline Total & 19 & 23 & 28 & 70 & 17 & 19 & 26 & 62 \\
\hline
\end{tabular}

${ }^{a, b}$ Superíndices diferentes dentro de géneros indica diferencia significativa $(p<0.05)$

Cuadro 3. $\mathrm{pH}$ del suelo en plantaciones de melina (Gmelina arborea) ubicados en la zona central del trópico húmedo ecuatoriano (2016)

\begin{tabular}{lcc}
\hline pH del suelo & 1 año & 3 años \\
\hline en agua & 6.23 & 6.45 \\
en $\mathrm{KCl}$ & 5.22 & 5.25 \\
\hline
\end{tabular}

de hospedero (Ramos y Guadarrama, 2004). Países amazónicos como Brasil y Colombia llevan entre 25 y 30 años investigando este tema y, en la actualidad, presentan importantes avances en relación con la distribución geográfica de las poblaciones de HMA en sus zonas geográficas (Stürmer y Siqueira, 2006).

Lara-Pérez et al. (2014) indican que existe una dinámica que identifica algunos consorcios de géneros de HMA en ecosistemas tropicales. Estos autores reportan que las comunidades de HMA pueden llegar a mantener una estrecha relación con el Bosque Húmedo Tropical y otros ecosistemas localizados en Centroamérica, con base a un estudio sobre colonización y estructura de la comunidad de HMA en bos- que mesófilo de montaña en Veracruz, México, donde encontraron hongos de los géneros Gigaspora, Acaulospora, Glomus, Funneliformis, Sclerocystis y Scutellospora.

La densidad de colonización visual en raíces de melina no presentó diferencia significativa entre las plantaciones de uno (1.9\%) y tres años $(1.7 \%)$ de edad. La densidad de colonización normalmente se debe a la asociación micorrízica que se ha registrado en el $90 \%$ de las plantas terrestres y que ha sido clasificada, según el grado de penetración de los hongos dentro de las raíces, en tres tipos: ectomicorriza, endomicorriza y ectendomicorriza (Guadarrama et al., 2004). De estos, un tipo particular de endomicorriza, la micorriza arbuscular, es la más abundante en los sistemas tropicales (Smith y Read, 2008).

El pH del suelo determinado en soluciones de agua $\left(\mathrm{H}_{2} \mathrm{O}\right)$ y de cloruro de potasio $(\mathrm{KCl})$ fue ligeramente ácido (Cuadro 3 ).

Las variables edafoclimáticas del lugar, independientemente de la edad, se ven favorecidas por la presencia de HMA. Sin embargo, diversos factores bióticos y abióticos afectan la colonización micorrizica en los ecosistemas; entre ellos el pH (Alvarado et al., 2004), el estrés hídrico (Gavito et al., 2008), la disponibilidad de luz (Shukla et al., 
2009) y la habilidad para obtener carbono producido por las plantas. Así, Alvarado et al. (2004) encontraron valores de $\mathrm{pH}<5.5 \mathrm{y}$ menos de $8 \%$ de colonizaciones micorrízicas en plantas de teca (Tectona grandis L) en Costa Rica.

\section{Conclusión}

El género Glomus es la especie prevalente en plantaciones forestales de melina (Gmelina arborea) en la zona central del trópico húmedo ecuatoriano.

\section{Literatura Citada}

1. Alvarado A, Chavarría M, Guerrero $R$, Boniche J, Navarro J. 2004. Características edáficas y presencia de micorrizas en plantaciones de teca (Tectona grandis L. f.). Agron Costrric 28: 89-100.

2. Brundrett M, Bougher N, Dell B, Grove T, Malajczuck N. 1996. Working whit mycorrhizas in forestry and agriculture. Camberra, Australia: ACIAR. 374 p.

3. Cuervo J, Rivas G. 2016. Cuantificación de hongos micorrícicos en muestras de suelo en plantaciones de Tabebuia rosea y Cordia alliodora. Nova 5(7): 38-41.

4. Donoso E, Lobos G, Rojas N. 2008. Efecto de Trichoderma harzianum y compost sobre el crecimiento de plántulas de Pinus radiata en vivero. Bosque 29(1): 52-57.

5. Gavito M, Pérez Castillo D, González Monterrubio C, Vieyra Hernández T, Martínez Trujillo M. 2008. High compatibility between arbuscular mycorrhizal fungal communities and seedlings of different land use types in a tropical dry ecosystem. Mycorrhiza 19: 47-60. doi: 10.1007/s00572-008-0203-4

6. Gerderman J, Nicholson T. 1963. Spores of mycorrihizal Endogene species extracted from soil by wet- sieving and decanting. T Brit Mycol Soc 46: 235-244.

7. Giovanotti M, Mosse B. 1980. An evaluation of techniques for measuring vesicular-arbuscular infection mycorrhizal in roots. New Phytol 84: 489-500. doi: 10.1111/j.1469-8137.1980.tb04556.x

8. Guadarrama P, Sánchez I, Álvarez J, Ramos J. 2004. Hongos y plantas, beneficios a diferentes escalas en micorrizas arbusculares. Ciencias 73: 38-45.

9. INVAM. (sf). (International Cultura Collection of (Vesicular) Arbuscular Mycorrhizal Fungi) Arbuscular mycorrhizal fungi. Key to fungi in glomales. [Internet]. Disponible en http:// fungi.invam.wvu.edu

10. Lara-Pérez LA, Noa Carrazana JC, Landa-López AJ, HernándezGonzález S, Oros-Ortega I, AndradeTorres A. 2014. Colonización y estructura de la comunidad de hongos micorrízicos arbusculares en Alsophila firma (Cyatheaceae) en bosque mesófilo de montaña en Veracruz, México. Rev Biol Trop 62: 1609-1623.

11. Méndez-Cortés H, MarmolejoMonsiváis J, Cantú-Ayala C, OlaldePortugal V, Estrada-Castillón E, Posadas-Leal C. 2013. Respuesta de Cedrela odorata L a diversos inoculantes micorrízicos procedentes de dos ecosistemas tropicales. Madera y Bosques 19(3): 23-34.

12. Peterson R, Massicotte H, Melvilla L. 2004. Mycorrhizas: anatomy and cell biology. Otawa, Canada: NRC Research Press. 173 p.

13. Phillips J, Hayman D. 1970. Improved procedures for clearing roots and staining parasitic and vasicular-arbuscular mycorrhizal fungi for rapid assessment of infection. T Brit Mycol Soc 55: 158161. doi: 10.1016/S0007-1536(70)80110-3

14. Prieto-Benavides O, BelezacaPinargote C, Mora-Silva F, GarcesFiallos R, Sabando-Ávila A, CedeñoLoja E. 2012. Identificación de hongos micorrízicos arbusculares en sistemas agroforestales con cacao en el Trópico 
Húmedo Ecuatoriano. Agron Mesoam 23(2): 233-239.

15. Ramos Zapata J, Guadarrama P. 2004. Los hongos micorrizógenos arbusculares en la restauración de comunidades tropicales. Universidad y Ciencia 1: 59-65.

16. [Reybampac] Rey Banano del Pacifico. 2012. Plan de manejo forestal. [Internet]. Disponible en: http://www.reysahi-wal.com/Resumen\%20Plan$\% 20$ de $\% 20$ Manejo\%20-Forestal.\%20Reybanpac\%20C.A..pdf

17. Ruíz P, Rojas K, Sieverding E. 2011. La distribución geográfica de los hongos de micorriza arbuscular: una prioridad de investigación en la amazonía peruana. Espacio y Desarrollo 23: 47-63.

18. SAS/STAT. 2002. SAS/STAT ${ }^{\circledR}$ Versión 9.0 Sistema SAS para Windows. Cary, NC, USA: SAS.

19. Shukla A, Kumar A, Jha A, Chatuveredi O, Prasad R, Gupta A. 2009. Effects of shade on arbuscular mycorrhizal colonization and growth of crops and tree seedlings in Central India. Agroforest Syst 76: 95-109. doi: 10.1007/s10457-008-9182-x

20. Smith S, Read D. 2008. Mycorrhizal symbiosis. $3^{\text {rd }}$ ed. USA: Academic Press. $800 \mathrm{p}$.
21. Steubing L, Godoy R, Alberdi M. 2001. Métodos de ecología vegetal. Santiago de Chile: Ed universitaria. $343 \mathrm{p}$.

22. Sturner SL, Siquerira O. 2006. Diversity of arbuscular mycorrhizal fungi in Brazilian ecosystems. En: Moreira FMS, Siqueira JO, Brussaard L (eds). Soil Biodiversity in Amazonian and other Brazilian ecosystems. Wallingford: CABI Publishing. p 206-236.

23. Trejo D, Ferrera $R$, Garcia $R$, Varela L, Lara L, Alarcón A. 2011. Efectividad de siste consorcios nativos de hongos micorrízicos arbusculares en plantas de café en condiciones de invernadero y campo. Rev Chil Hist Nat 84: 2331. doi: 10.4067/S0716-078X2011000100002

24. van der Heijden M, Klironomos JN, Ursic M, Moutoglis P, Estreitwolf R, Boller T, Wiemken A, Sanders I. 1998. Mycorrhizal fungal diversity determines plant biodiversity, ecosystem variability and productivity. Nature 396: 69-72. doi: 10.1038/23932

25. Varela L, Trejo D. 2001. Los hongos micorrizógenos arbusculares como componentes de la biodiversidad del suelo en México. Acta Zool Mex (núm esp 1): 39-51. 